\title{
Anabases
}

ANABASES Traditions et réceptions de l'Antiquité

$7 \mid 2008$

Varia

\section{Expérience et méthode : introduction à un entretien avec Jean-Pierre Vernant}

José Otávio Guimarães

\section{OpenEdition}

1 Journals

Édition électronique

URL : http://journals.openedition.org/anabases/2438

DOI : 10.4000/anabases.2438

ISSN : 2256-9421

Éditeur

E.R.A.S.M.E.

Édition imprimée

Date de publication : 1 mars 2008

Pagination : 11-16

ISSN : 1774-4296

\section{Référence électronique}

José Otávio Guimarães, « Expérience et méthode : introduction à un entretien avec Jean-Pierre Vernant », Anabases [En ligne], 7 | 2008, mis en ligne le 01 décembre 2011, consulté le 21 octobre 2019. URL : http://journals.openedition.org/anabases/2438 ; DOI : 10.4000/anabases.2438

(c) Anabases 
Anabases 6 (2007), p. 11-16.

\section{Expérience et méthode : introduction à un entretien avec Jean-Pierre Vernant}

José OTÁVIO Guimarães

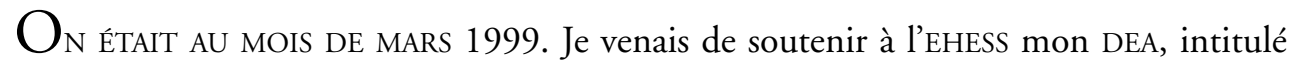
"Jean-Pierre Vernant polumètis : les ruses d'une anthropologie de la Grèce antique ». Je débutais donc mon doctorat, lorsque François Hartog, mon directeur de thèse au Centre Louis Gernet de recherches comparées sur les sociétés anciennes, m’a dit sans hésiter : "C'est l'heure d'aller rencontrer Vernant ${ }^{1}$. "Travaillant dans le domaine de l'histoire intellectuelle contemporaine, il fallait en effet commencer à élaborer mes sources. Après avoir établi dans le mémoire un premier questionnaire historiographique et avoir fixé mes repères conceptuels, je devais me plonger dans la recherche des témoins. Ainsi, en donnant suite à mon enquête sur la formation et la transformation de la notion d'anthropologie historique dans le milieu des hellénistes français de la seconde moitié du XXe siècle, en me penchant particulièrement sur les dialogues et les silences, les échanges et les blocages, entre Vernant et les historiens de la mouvance des Annales - où la notion, on le sait, a trouvé un terrain fertile en tant qu'une des expressions du compromis tendu entre histoire et sciences sociales dans les années 1960 et 1970 -, il m’a semblé fondamental d'interroger l'auteur de Les origines de la pensée grecque (1962) à propos de ses rapports personnels et intellectuels avec trois des noms les plus importants des sciences humaines françaises : Georges Dumézil, Claude Lévi-Strauss et Michel Foucault. Les domaines d'étude de ces trois personnages - la linguistique, l'anthropologie et la philosophie (bien que ces étiquettes ne rendent pas compte de la dimension pluridisciplinaire de leurs ouvrages) - renforçaient la perspective par laquelle

1 Je tiens à remercier François Hartog, à qui je dois non seulement mon séjour de recherches en France, mais aussi la possibilité d'avoir connu personnellement Jean-Pierre Vernant. Merci aussi à la CAPES et à l'Université de Brasília pour les bourses qu'elles m’ont accordées. 
on soulignait la contribution majeure de Vernant aux études contemporaines sur la Grèce ancienne, à savoir sa position au carrefour des diverses branches des " humanités ". Les trois personnages ont été collègues de Vernant dans la " marginalité prestigieuse 2 " du Collège de France ; les deux premiers ont en outre joué un rôle très important afin que l'helléniste y soit reçu, en 1974, après avoir été élu à la chaire d'Études comparées des religions antiques 3 . C'est au Collège d'ailleurs que, comme professeur honoraire, Vernant m'a accueilli, le 14 avril 1999, pour ce premier entretien.

Traiter des rapports intellectuels de Vernant avec Dumézil et Lévi-Strauss n'est pas une nouveauté. Bien souvent, dans des textes publiés de son vivant, l'helléniste a reconnu ses dettes envers l'un et l'autre ${ }^{4}$. Ce qui me semble original dans les opinions soutenues par Vernant dans cette conversation est l'exploitation du problème singulier du rapport au temps historique chez les trois collègues, dieux indiscutés de ce qu'il est convenu d'appeler, de manière certes imprécise, le panthéon structuraliste. Vernant a fait, on le sait, un usage bien pragmatique de la contribution structurale, en se servant d'une certaine méthode d'analyse du récit sans accepter pourtant les implications philosophiques d'une pensée de la structure. En ce sens, ce qu'il dit sur Foucault peut être ici du plus haut intérêt. Parmi les trois collègues, le philosophe est celui qui apparaît le moins dans les écrits de l'anthropologue de la Grèce ancienne. Vernant, d'ailleurs, n'a jamais caché son inconfort face à certaines thèses de Les mots et les choses - ce livre qui, selon François Furet, a systématisé, dans le feu de l'action, la " coupure épistémologique " représentée par l'ethnologie structurale par rapport à "l'âge idéologique 5 ". D'autre part, Vernant s'est montré moins résistant au Foucault des derniers livres, l'Histoire de la sexualité. Il a constaté dans la posture de l'antiquisant néophyte des résonances de la démarche historiographique de son anthropologie de la Grèce ancienne. Du reste, Foucault lui-même, en précisant, en 1973, sa notion de discours par rapport à la conception de la philosophie analytique anglo-saxonne et à la perspective fondée sur la linguistique de Saussure, a évoqué justement la conduite de Dumézil et Vernant. Les deux, soutient Foucault, pensent que des rapports structuraux peuvent « intervenir

2 L'expression est de J. REVEL, "Histoire et sciences sociales : une confrontation instable", in J. Boutier et D. Julia (dirigé par), Passés recomposés : champs et chantiers de l'Histoire, Paris, Autrement, 1995, p. 75.

3 Vernant a raconté, en des termes proches de ceux de l'entretien, les aléas de cette élection dans La traversée des frontières, Paris, Le Seuil, 2004, p. 63-64.

4 Il suffit de se référer à "Histoire et structure dans la religion romaine archaïque ", in Religions, histoires, raisons, Paris, François Maspero, 1979, p 35-53 ; "Les raison du mythe”, in Mythe et sociéte en Grèce ancienne, Paris, La Découverte, 1988, p. 195-250 ; "La religion objet de science" et "Le mythe au réfléchi", in Entre mythe et politique, Paris, Le Seuil, 1996, p. 94-104 et 352-356.

5 F. FuRET, "Les intellectuels français et le structuralisme", Preuves, n. 92, février 1967 ; repris in L'atelier de l'histoire, Paris, Flammarion, 1982, p. 46. 
aussi bien dans un discours que dans un rituel religieux ou dans une pratique sociale ». Loin donc d'identifier ou de projeter "toutes les structures sociales, les pratiques sociales, les rites, dans un univers du discours ", ils replacent, au fond, "la pratique du discours à l'intérieur des pratiques sociales ${ }^{6}$ ". Enfin, c'est dans les recherches de l'helléniste et de son groupe que Foucault voit le déploiement méthodologique du travail initié par le linguiste : «Il y a actuellement en France un groupe autour de Jean-Pierre Vernant qui reprend un peu les idées de Dumézil et essaie de les appliquer ${ }^{7}$."

En parlant de ses rapports avec les trois savants, des questions sensibles et difficiles sont apparues, et Vernant les a affrontées avec limpidité et courage. Il a, par exemple, refusé les liens entre certaines positions politiques et idéologiques de Dumézil et ses recherches sur la mythologie indo-européenne. Du plan politique au plan intellectuel les chemins sont tortueux et minés, et ils doivent être parcourus avec beaucoup de prudence et d'habileté. « Je ne crois pas du tout, nous dit Vernant, que le travail scientifique et que les positions que prend un savant peuvent s'expliquer par ses positions politiques. En tout cas, elles n'en sont pas le reflet, elles ont une autonomie. » Il est même surprenant qu'après s'être exprimé en ces termes, Vernant ait tout de suite souligné les différences politiques qui le séparent du linguiste : "Il n'était pas proche des positions qui pouvaient être les miennes ; [...] il avait parfaitement le droit d'être de l'Action française, un nationaliste conservateur. " Le même genre de problème émerge plus loin quand Vernant raconte l'épisode de la tentative de publication aux États-Unis de son Mythe et pensée chez les Grecs. Dans ce cas, le relativisme méthodologique de l'anthropologue-historien est explicite : on ne peut pas considérer le portrait de Pandore peint par Hésiode comme du machisme moderne ; en d'autre termes, il n'est pas possible d'user d'un poète grec archaïque, dont les vers remontent à plus de deux millénaires, pour appuyer la cause politique d'un féminisme aux prétentions gauchistes. Même si la procédure de l'historien sérieux se révèle aussitôt bien visible (on ne doit pas se faire piéger par l'anachronisme), une certaine subjectivité vernantienne ainsi que les dilemmes de son présent font également tout à coup surface. Il n'est pas facile d'ignorer l'antiaméricanisme qu'a gardé le vieil ex-militant communiste ou encore les difficultés qu'il a eues - dont il s'est même rendu compte - d'aborder les questions de la sexualité ou des études de genre posées par les derniers travaux de Foucault et développées par quelques chercheuses du Centre Louis Gernet. Ces questions deviennent d'autant plus sensibles et délicates quand on s'aperçoit que Vernant, en échappant à l'identification immédiate des positions politiques et idéologiques de Dumézil avec les analyses de la mythologie indo-européenne, suggère des hypothèses alternatives pour

6 M. FOUCAULT, "La vérité et les formes juridiques" (Conférences à l'Université pontificale catholique de Rio de Janeiro, du 21 au 25 mai 1973), in Dits et écrits I, 1954-1975, Paris, Gallimard, coll. Quarto, 2001, p. 1504. 
expliquer la caractérisation du monde arien faite par le linguiste, à savoir, son orientation homosexuelle ou le fait d'avoir eu un père militaire. L'observation rappelle les troublants rapports de Vernant à la psychanalyse, pour laquelle la sympathie de Foucault ne lui a pas échappé. Hélas, le sujet n’a pas pu être développé par Vernant à l'occasion de cette rencontre, sauf lorsqu'il a signalé que les psychanalystes n'avaient pas pour lui grande estime ${ }^{8}$.

L'expression "comme une barque sur un fleuve", choisie comme titre du texte, est de Vernant lui-même et a été extraite de l'entretien. Dans son contexte d'énonciation, elle se référait au mouvement de transformation permanente qui s'empare de toute entreprise de recherche déployée pendant un temps plus ou moins long. L'helléniste s'en est également servi pour exprimer l'idée du contrôle limité que détient tout savant sur la direction et les détours de sa démarche d'investigation. Ainsi, interrogé sur l'impact que les travaux de Lévi-Strauss ont pu exercer, à partir d'un certain moment, sur ses analyses du mythe grec, Vernant, en essayant de relativiser les déterminismes simplistes fondés sur les notions d' "influence " appartenant à une vieille histoire des idées, a répondu : "Quand on suit une recherche, on est comme une barque sur un fleuve. Il y a, à la fois, le mouvement de la barque et des rames et puis le fleuve qui vous entraîne. "

Certains points soulevés par Vernant lors de l'entretien peuvent bien être pris comme des apports aux relations entre expérience et méthode que lui-même n’a cessé d'explorer durant les dernières années de sa vie. "Il n'est pas absurde " - écrit Reinhart Koselleck dans un texte consacré à ces relations - "d'établir un lien entre les interventions méthodologiques des historiens et les expériences tout à fait personnelles par lesquelles ils ont un jour été concernés et qui sont indispensables pour comprendre leur innovations 9." Dans son exercice strict du relativisme méthodologique, Vernant a résisté pendant une longue période de sa vie savante à la tentation de développer ces relations. Toutefois, il reconnaît avoir, à partir d'un certain moment, été " sensible ", par exemple, à quelques thèmes et personnages du passé parce qu'il avait lui aussi vécu tout au long de sa vie des expériences similaires. Interrogé, lors du lancement de L'individu, la mort, l'amour, en 1989, sur le fait que le livre rappelait « dans une certaine mesure des préoccupations contemporaines " — il a répliqué : «Voilà pourtant un débat, dont je ne nie pas qu'il existe, que j'ai toujours écarté, pour des raisons méthodologiques, ou d'éthique de recherche. Que j'évite moins aujourd'hui, parce que je suis devenu vieux,

8 Sur Vernant, Foucault et la psychanalyse, voir M. LEONARD, "Oedipus and the Political Subject", in Athens in Paris - Ancient Greece and the Political in Post-War French Thought, Oxford, Oxford University Press, 2005, p. 22-95.

9 R. KOSELLECK, "Mutation de l'expérience et changement de méthode", in L'expérience de l'histoire, trad. fr. de A. Escudier (avec la collaboration de D. Meur, M.-C. Hoock et J. Hoock), Paris, Hautes Études-Gallimard-Le Seuil, 1997, p. 208. 
et peut-être parce que je me laisse plus aller aux confidences ${ }^{10} \ldots$ ». Il avait alors 75 ans. Il est vrai que, deux ans après, dans l'introduction qu'il a écrite pour L'homme grec, il s'excusait d'y faire usage d'un exemple personnel; mais il le faisait quand même ${ }^{11}$. Lors de notre entretien, il avait déjà 85 ans. En fait, si l'on considère la durée d'une vie, le vieillissement, ou tout simplement la modification de la position du présent par rapport à la naissance et la mort, change le jeu et les tensions entre l'espace d'expérience et l'horizon d'attente. Cela ne signifie pas que ce changement a conduit Vernant à se laisser séduire par l'illusion biographique, ou plutôt autobiographique, alors que la montée de la notion de mémoire dans les années 1980 et 1990 suscitait l'intérêt d'un public plus large pour ce genre de littérature. Bien au contraire, dans la préface de Entre mythe et politique, il remarquait ses difficultés à tenir la plume autobiographique. La même observation est reprise au début de La traversée des frontières, où il affirme que l'écriture mémorialiste lui semblait entièrement " étrangère " à ses « inclinations " et à ses " capacités 12 ». Les confidences seraient utiles à l'helléniste, comme il le fait de manière plus systématique dans ce dernier ouvrage, pour entamer " une réflexion générale qui dépasse largement [sa] personne 13 ». Cette réflexion générale, pour la joie de ceux qui s’intéressent à l'expérience de l'histoire que nous ont léguée la vie et l'œuvre de cet helléniste, concerne les " rapports du passé et du présent, les frontières qui les séparent, les moyens de franchir ces limites sans les brouiller, sans les fausser ${ }^{14}$ \#.

Je garde encore vivantes en mémoire la générosité et la grandeur de cet intellectuel. Je n'oublie pas la rapidité avec laquelle il a invariablement répondu à mes sollicitations. Il me téléphonait immédiatement afin de définir les dates de nos rencontres et en profitait pour commenter les questions que je lui envoyais. Je me souviens que, pour ce premier rendez-vous, il m'a attendu dans le hall du Collège et m'a accompagné ensuite jusqu'à son bureau. Dès le début, il m’a demandé de laisser tomber le vous et de le tutoyer. J'ai bien essayé, mais je n'y suis pas parvenu. Ensuite, lorsque j’ai sorti mes papiers avec les questions que j'avais préparées, il a réagi immédiatement en me disant qu’on pouvait engager une conversation plus informelle. J'ai cédé malgré l'inquiétude de ne pouvoir me référer à mes notes. En fait, elles n'étaient pas nécessaires, puisqu'il

10 J.-P. VERnANT, “Anthropologie historique et Grèce ancienne”, Raison présente 91 (1989), p. 127.

11 «Un exemple pour me faire comprendre, dont on pardonnera ce qu'il comporte de personnel : comment pourrions-nous voir aujourd'hui la lune avec les yeux d'un Grec ? J'ai pu m’y essayer du temps de ma jeunesse, à mon premier voyage en Grèce. " "Introduction", in L'homme grec (sous la dir. de J.-P. VERNANT), Paris, Le Seuil, 1993, p. 9.

12 VERNANT, La traversée de frontières, p. 9.

13 Ibid.

14 Ibid. Concernant cette réflexion générale de Vernant, voir le bel article de P. PAYEN, "Par delà les frontières. L'héritage politique grec dans les œuvres de Jean-Pierre Vernant et Pierre Vidal-Naquet" (à paraître). 
suffisait de lancer une idée, de proposer un sujet pour que Vernant se mette à parler. Il était difficile de l'interrompre, et là n'était pas mon but. Son discours était généreux, net, impatient ; il mettait en jeu non seulement le mouvement vigoureux de ses mains mais aussi celui de tout son corps. Je me souviens bien qu'il vibrait dans son fauteuil. Je l'écoutais, quant à moi, avec une vibrante émotion.

José Otávio GuIMARÃES

Centre d'études classiques

Département d'Histoire

Université de Brasilia

nogueira@unb.br 